\title{
Role of Yes-associated protein in cancer: An update (Review)
}

\author{
RAMAZAN ABYLKASSOV and YINGQIU XIE
}

Department of Biology, Nazarbayev University School of Science and Technology, Astana 010000, Republic of Kazakhstan

Received April 6, 2015; Accepted June 21, 2016

DOI: $10.3892 / 01.2016 .4955$

\begin{abstract}
Yes-associated protein (YAP) is an oncoprotein located in the cytoplasm in an inactive form, and when activated, it translocates to the nucleus and activates the transcription of genes responsible for cell division and apoptosis. YAP is one of the downstream regulatory proteins in the Hippo signaling pathway, which is important in cell proliferation and regeneration. Due to its great importance, YAP is regulated very strictly by different regulatory systems. The present review will focus on the canonical pathways of YAP, and will provide details on the most recent findings regarding its regulation and role in tumorigenesis, specifically in prostate tumor progression.
\end{abstract}

\section{Contents}

1. Introduction

2. Structural domains of YAP protein

3. Regulation of YAP

4. Function of YAP in cancer

5. Implications for clinical targeted therapy

\section{Introduction}

Yes-associated protein (YAP or YAP1) is an oncoprotein encoded by the YAP gene in the human chromosome 11q22 (1). YAP is one of the downstream proteins in the Hippo signaling pathway (1). It functions in cooperation with transcriptional coactivator with PDZ-binding motif (TAZ) (2). These two proteins are responsible for cell proliferation control and have important regulatory functions in regeneration, organ development and stem cell self-renewal. YAP and TAZ both are regulated by several mechanisms, including the microenvironment and extracellular signals. YAP is also able to inhibit

Correspondence to: Dr Yingqiu Xie, Department of Biology, Nazarbayev University School of Science and Technology, 53 Kabanbay Batyr Avenue, Astana 010000, Republic of Kazakhstan E-mail: xieautumnus@gmail.com

Key words: YAP, cancer, prostate cancer apoptosis in cells through the activation of survival signaling such as survivin/baculoviral IAP repeat containing 5 (2). Recent investigations suggest that YAP crosstalks with numerous signaling pathways and has more functions in cancer than previously assumed.

\section{Structural domains of YAP protein}

YAP consists of 488 amino acids and several structural domains (3) (Fig. 1). The most important domains are a TEA DNA-binding domain and two WW domains (2). The first one binds to the TEA domain (TEAD) family of transcription factors, while the second one binds to a transcriptional coactivator, which in turn, binds to the PPxY motif present on transcription factors (4). In addition, the PDZ-binding motif regulates the function of YAP by localizing YAP to the specific nuclear foci and is essential for YAP-mediated cellular transformation (5). The SRC homolog 3-binding motif regulates the activity of YAP through binding to p53 binding protein 2, in cooperation with the WW1 domain (6).

\section{Regulation of YAP}

The regulation of YAP occurs mostly through the regulation of the Hippo signaling pathway (2). Large tumor suppressor 1 (LATS1) and LATS2 are the inhibitors of the YAP/TAZ complex. When activated, these two proteins phosphorylate YAP and localize it in the cytoplasm (2). YAP is tightly regulated by multiple factors, including extracellular signals and the microenvironment (5).

Regulation by extracellular signals. One of the most important regulators of YAP is G-protein coupled receptor (GPCR), which consists of three subunits, $\alpha, \beta, \gamma$, among which, $G \alpha_{11}$, $\mathrm{G} \alpha_{12}, \mathrm{G} \alpha_{13}, \mathrm{G} \alpha_{\mathrm{i}}, \mathrm{G} \alpha_{\mathrm{o}}$ and $\mathrm{G} \alpha_{\mathrm{q}}$ activate YAP and TAZ, while $\mathrm{G} \alpha_{\mathrm{s}}$ inhibits YAP/TAZ activity (7). GPCR activates YAP by inducing F-actin polymerization through Rho GTPase, and thus inhibit LATS kinase activity (7). There are two types of ligands-agonists that activate GPCR: i) Lipoprotein(a), sphingosine-1-phosphate and thrombin, which inhibit cell proliferation; and ii) glucagon and epinephrine, which induce cell proliferation (8) (Fig. 2). In addition, Wnt signaling can regulate YAP activity by separating YAP from the $\beta$-catenin destruction complex, and thus causing accumulation of nuclear YAP (Fig. 2) (9). Leukemia inhibitory factor receptor and epidermal growth factor can activate YAP by dissociating it from Hippo kinase (7). 


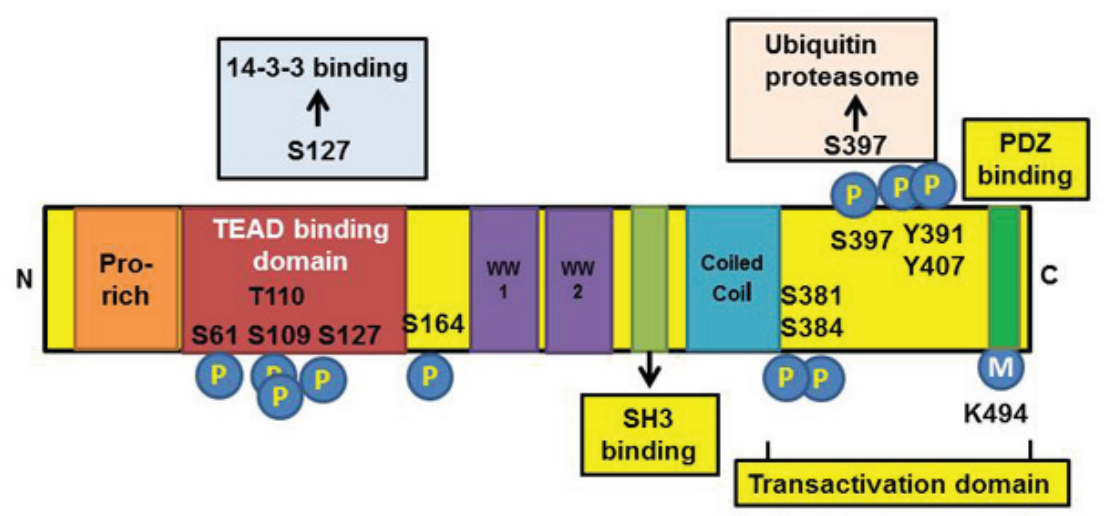

Figure 1. YAP protein domains. Various domains of YAP are colored from the N-terminus to the C-terminus, highlighting phosphorylation and methylation sites, as well as binding sites with other factors. Figure is adapted from Zhao et al (3). YAP, yes-associated protein; TEAD, TEA domain; SH3, SRC homology 3; P, phosphorylation; M, methylation.

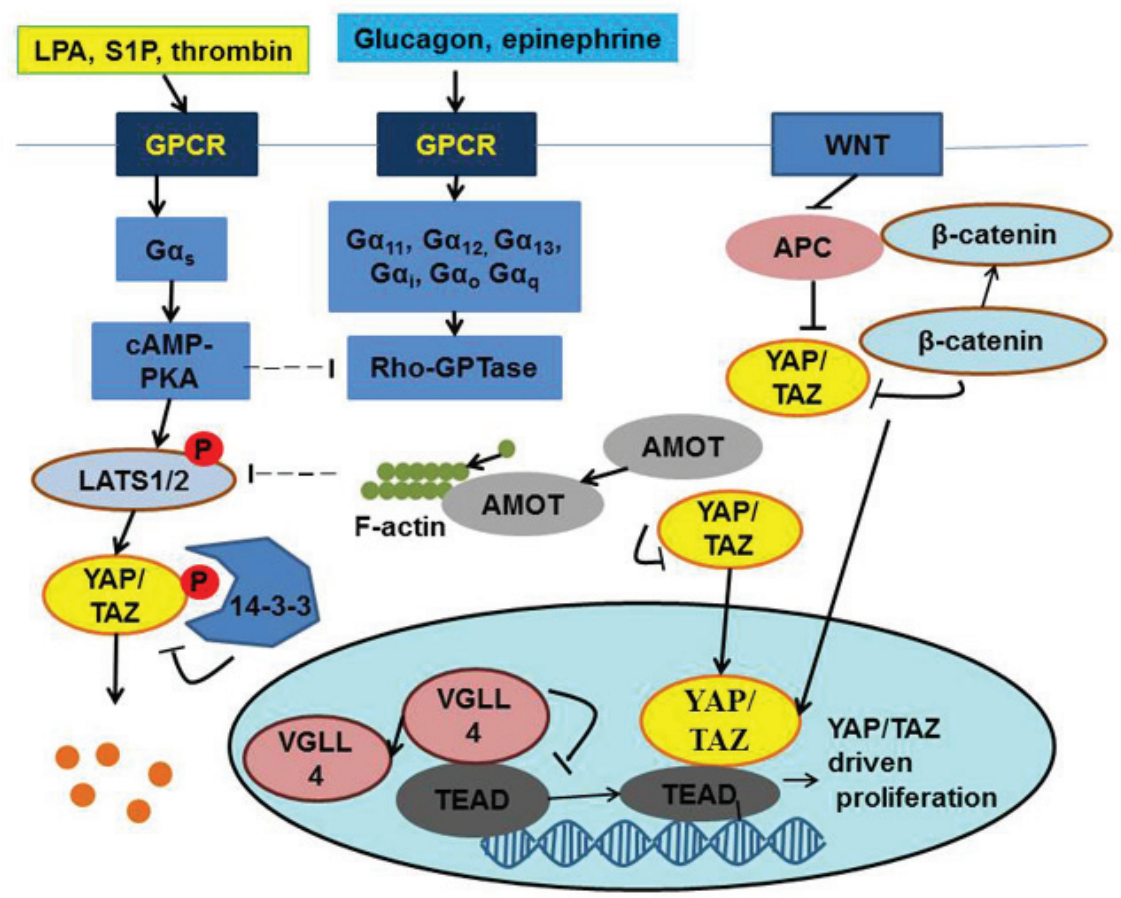

Figure 2. Regulation of the Yes-associated protein/transcriptional coactivator with PDZ-binding motif complex. YAP, Yes-associated protein; TAZ, transcriptional coactivator with PDZ-binding motif; GPCR, G-protein-coupled receptor; LPA, lipoprotein(a); S1P, sphingosine-1-phosphate; APC, adenomatous polyposis coli; cAMP, cyclic adenosine monophosphate; PKA, protein kinase A; LATS, large tumor suppressor; AMOT, angiomotin; VGLL4, vestigial-like family member 4; TEAD, TEA domain; P, phosphorylation.

Regulation by microRNA (miRNA). Increasing evidence suggest that miRNAs regulate YAP, including miRNA 31, which is considered as an oncogene that acts through YAP during cancer progression (10). The overexpression of miRNA 31 leads to anchorage-independent uncontrolled growth in vitro and increases the potential of tumor formation in vivo (10). The expression level of miRNA 31 was much more increased in patients with high risk of recurrence, compared with those with low risk of recurrence (10). According to Mitamura et al (10), miRNA 31 suppresses the luciferase activity of messenger (m)RNA combined with LATS2 3' untranslated region (UTR). This promoted an increase in the nuclear level of YAP and moreover, enhanced the transcription of oncogenes such as cyclin D1.
Regulation by phosphorylation and methylation. Phosphorylation is the main pathway to inhibit YAP activity (7). As shown in Fig. 1, multiple sites of YAP can be phosphorylated. Nuclear Dbf2-related/LATS kinases regulate YAP1 through its phosphorylation at Serine 127, and promotes its exclusion from the nucleus into the cytoplasm and its degradation (11). Consequently, the transcription of pro-growth genes is inhibited. The artificial depletion of these kinases in the colon results in the formation of cancer (11). The phosphorylation of YAP at Serine 127 promotes binding of 14-3-3 protein to YAP and, thus, localizes it in the cytoplasm (4). Another kinase that promotes binding of 14-3-3 protein to YAP is Akt kinase (12). The inhibition of YAP by Akt causes inhibition of transcription factors, including $\mathrm{p} 53$, which regulates the pro-apoptotic 


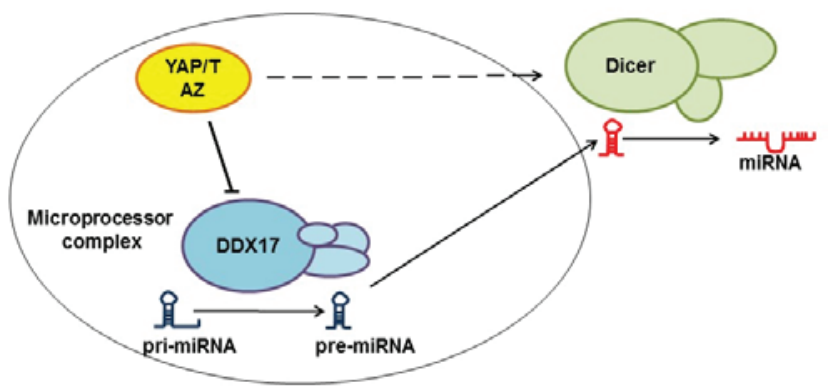

Figure 3. Regulation of microRNA synthesis by the Yes-associated protein/ transcriptional coactivator with PDZ-binding motif complex. pri-, primary transcript; pre-, precursor; miRNA, microRNA; YAP, Yes-associated protein; TAZ, transcriptional coactivator with PDZ-binding motif; DDX17, DEAD box helicase 17.

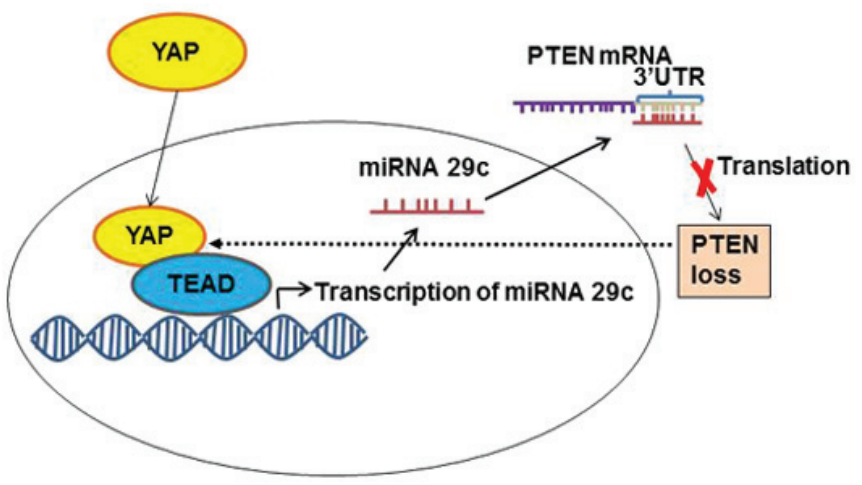

Figure 4. Regulation of the tumor suppressor phosphatase and tensin homolog by Yes-associated protein. TEAD, TEA domain; YAP, Yes-associated protein; miRNA, microRNA; mRNA, messenger RNA; PTEN, phosphatase and tensin homolog; UTR, untranslated region.

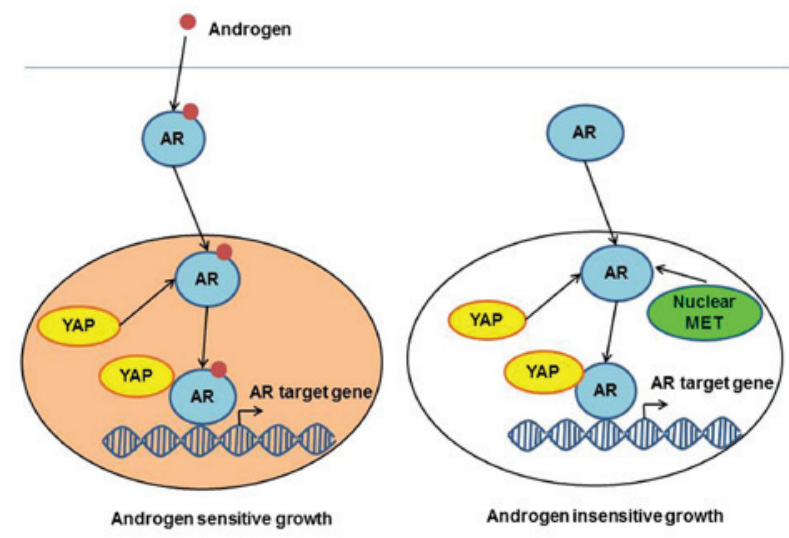

Figure 5. Function of YAP in prostate cancer cells. Upregulated activity of YAP causes the cells to become insensitive to androgen, and promotes uncontrolled expression of androgen receptor-dependent genes. YAP, Yesassociated protein; AR, androgen receptor.

gene B-cell lymphoma (Bcl)-2 associated $\mathrm{X}$ protein. Thus, the phosphorylation and consequent inhibition of YAP by Akt suppresses the induction of pro-apoptotic gene expression upon cell damage (12). Another molecule that regulates YAP function through phosphorylation is protein kinase $\mathrm{C} \xi$ $(\mathrm{PKC} \zeta)$, which plays an important role in the retention of YAP in the cytoplasm (13). PKC $\zeta$ phosphorylates YAP at Serine 109 and Threonine 110, which are highly conserved residues among different species (13). In leucine-rich repeat-containing G-protein coupled receptor 5-positive intestinal stem cells, the inactivating mutation of YAP causes increased tumorigenic and regenerative activities (13).

YAP methylation is another regulatory mechanism that localizes YAP in the cytoplasm and inhibits its function (14). The methylation of YAP is induced by $\mathrm{Su}(\mathrm{var}) 3-9$ and enhancer of zeste (SET)7, a SET-domain-containing lysine methyltransferase. Monomethylation of YAP at Lysine 494 occurs in parallel with YAP phosphorylation at Serine 127, which promotes the cytoplasmic localization of YAP (15) (Fig. 1).

Regulation by metabolism. The sterol regulatory elementbinding protein/mevalonate signaling pathway, an important cellular metabolic pathway, is able to regulate the activity of YAP. According to Sorrentino et al (16), the inhibition of its rate-limiting enzyme (3-hydroxy-3-methylglutaryl-coenzyme A reductase) opposes YAP/TAZ nuclear localization. The production of geranylgeranyl pyrophosphate generated by the mevalonate pathway is required for the activation of Rho GTPase (16). This GTPase is responsible for the polymerization of F-actin, which removes angiomotin (AMOT) from its complex with YAP, and thus allows YAP to translocate to the nucleus and to induce the transcription of pro-growth genes (7).

Regulation by the microenvironment. The microenvironment plays an important role in the regulation of YAP activity. Recent studies revealed that the stiffness of the extracellular matrix can cause changes in the levels of YAP in the nucleus (17). When the cells are placed into soft matrix, the cytoplasmic level of YAP increased and the cell proliferation rate decreased, and vice versa, when the cells were subjected to a stiff environment, the cytoplasmic YAP moved to the nucleus and induced cell proliferation (18). When cells undergo pressure, to balance the tension, they create an opposite force with the help of the cytoskeleton (17). Thus, when F-actin polymerization happens, the nuclear level of YAP increases and the cells start to proliferate (17). According to Mana-Capelli et al, AMOT links these two processes (19). AMOT is an inhibitor of YAP, and can act on it either directly or through activating LATS1 and LATS2 (19). When F-actin polymerization occurs, it competes with YAP for the binding to AMOT, and thus weakens the inhibitory effect of AMOT on YAP (18). Additionally, F-actin is also able to suppress the activity of LATS1/2, and thus further increases the nuclear level of YAP (18).

\section{Function of YAP in cancer}

As an oncoprotein, YAP controls transcription factors that regulate cell division and cell cycle, such as TEAD family members (7). TEAD proteins are associated with the transcription cofactor vestigial-like protein 4 (VGLL4) in steady state, which suppresses target gene expression (20). When the Hippo signaling pathway is activated, YAP is phosphorylated, which prevents YAP from passing through the nuclear pores and reaching TEAD proteins (20). When the pathway is inactivated, YAP moves to the nucleus, displaces VGLL4 from TEAD proteins and initiates cell division. In numerous 


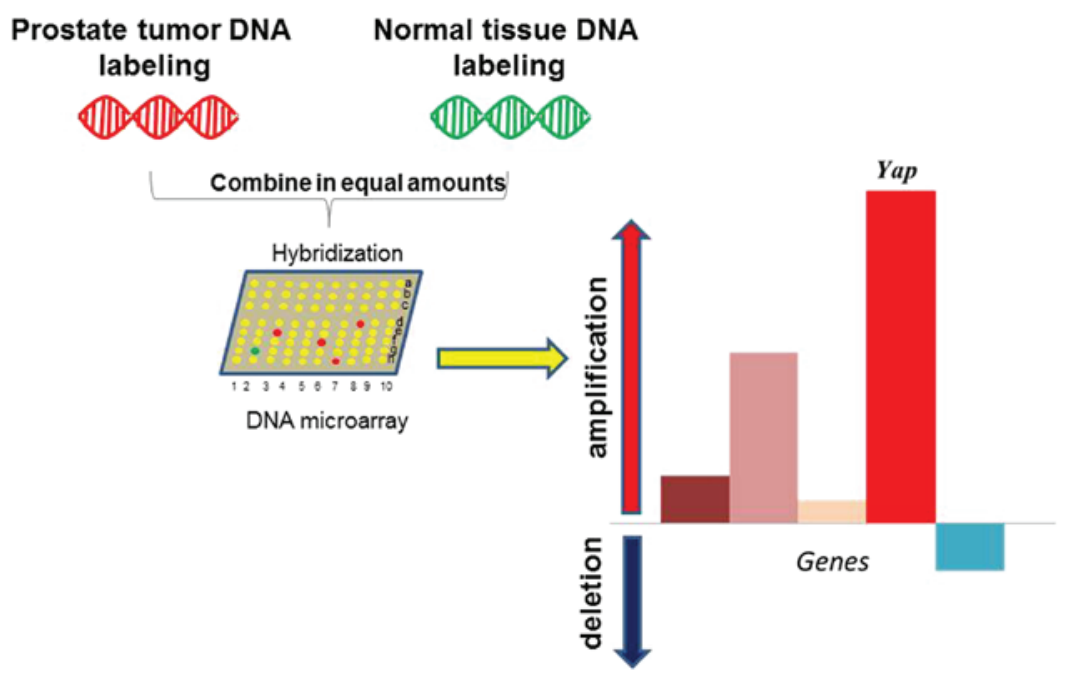

Figure 6. Array comparative genomic hybridization identified an elevation in the copy number of the mouse Yap gene in a mouse model of prostate cancer. The array comparative genomic hybridization method was used to determine the elevated or inhibited expression of several molecules. The Yap gene was highly amplified, and was one of the top ranked amplified genes (33). Yap, Yes-associated protein.

cancers, including hepatocellular carcinoma (HCC), ovarian cancer and non-small cell lung cancer, elevated nuclear level of YAP was observed, suggesting an association between YAP function and uncontrolled cell division (21).

Regulation of miRNA biogenesis. The suppression of miRNAs has been proposed to promote tumorigenesis (22). In order for miRNAs to become functional, they should first be subjected to the Microprocessor and Dicer complexes in the form of primary miRNA transcripts. Recent studies have demonstrated that YAP regulates miRNA production through diverting DEAD box helicase 17 (DDX17) from the Microprocessor complex in a cell-density dependent manner. When the cell density is low, YAP localizes in the nucleus and removes DDX17 from the Microprocessor complex, and when the cell density is high, YAP moves to the cytoplasm and DDX17 becomes free to combine with the Microprocessor complex (22). However, nuclear YAP/TAZ also regulates Dicer complexes, and thus mediates the formation of specific miRNAs (23). In summary, the YAP/TAZ complex may have opposite regulatory effects on different miRNAs (Fig. 3).

Regulation of phosphatase and tensin homolog (PTEN). PTEN, as a tumor suppressor protein, inhibits the phosphatidylinositol 3-kinase-mechanistic target of rapamycin signaling pathway, and is responsible for controlling organ size and cell proliferation (24). YAP regulates this pathway through PTEN. YAP inhibits PTEN activity by activating transcription factors of the TEAD family to express miRNA 29c (24) (Fig. 4). This miRNA 29c regulates PTEN function by targeting PTEN 3'UTR (24). When the level of activated YAP is increased, the level of PTEN decreases, and vice versa, when YAP is phosphorylated and inhibited, PTEN function is restored and oncogene transcription is inhibited (24). Therefore, mutations in the Hippo signaling pathway, which lead to an increase in the nuclear level of YAP, also cause the inhibition of its natural tumor suppressor, PTEN, and thus facilitate tumor formation.
Regulation of senescence. YAP is able to control senescence tightly through its activity. According to Fausti et al (25), the accumulation of YAP correlates with slower cell division rate and accelerated senescence. The authors stated that the loss of Werner syndrome ATP-dependent helicase protein activity in Werner syndrome activates the ataxia telangiectasia mutated (ATM)-YAP-promyelocytic leukemia protein (PML)-p53 axis, and thus induces the senescence process. ATM kinase is required for YAP-PML complex formation, and this complex promotes p53 activation (25). However, according to Xie et al (26), the senescence process of the cell is caused by downregulation of YAP levels, and the silencing of the YAP gene causes inhibition of cell proliferation and induces senescence. According to that report, YAP regulates the expression of cell division protein kinase $6(\mathrm{Cdk} 6)$, an important protein in the regulation of cell cycle progression. The results demonstrated that introduction of either YAP or Cdk6 into YAP-knocked down cells inhibited senescence and restored the proliferative abilities of the cells (26). In summary, there are different points of view about the regulation of cell senescence, and further studies are required to clarify this mechanism.

Regulation of Ras-dependent oncogenesis. Overexpression of proteins from the Ras family leads to uncontrolled cell growth and cancer, such as pancreatic ductal adenocarcinoma (27). Elevated level of YAP was observed in K-Ras suppression-resistant cancer cells (28). Recently, YAP was identified as the main compensator of cell proliferation for loss of K-Ras signaling in K-Ras-dependent cancers (29). K-Ras and YAP proteins converge on the transcription factor FOS, and activate the transcription of the genes responsible for the regulation of the epithelial-mesenchymal transition, which initiates metastasis in cancer cells (28).

YAP function in prostate cancer progression. Androgen receptor (AR), as a transcription factor, plays a central role in prostate cancer progression through regulating the 
transcription of cell proliferation-related genes. Recent studies have shown that YAP is able to bind and activate nuclear AR, thus promoting cell proliferation (30). Binding of AR to YAP occurs via the PDZ binding domain and the coiled-coiled protein-protein interaction domain (30). The YAP complex can activate androgen-dependent transcription of AR target genes, which has the same DNA binding site. However, enhanced level of YAP mRNA was observed in androgen-insensitive prostate cancer cells, compared with androgen-sensitive cells (31). Furthermore, immunohistochemistry revealed increased levels of activated YAP in castration-resistant prostate tumors, compared with hormonal-responsive prostate tumors (31). Further studies demonstrated that YAP overexpression was sufficient to cause the transition of cells from an androgen-sensitive to an androgen-insensitive phenotype in vitro, and YAP conferred castration resistance in vivo (31). Extracellular signal-regulated kinase ribosomal S6 kinase signaling appeared to be regulated by YAP in cell survival, migration and invasion in androgen-insensitive cells, and thus, the downregulation of YAP in prostate cancer cells greatly reduced their migratory and invasive rates, and in androgen-deprivation conditions, it inhibited cell division (31). In summary, in prostate cells with deregulated Hippo signaling pathway, YAP can promote cancer cells to lose their sensitivity to androgen and transform into uncontrolled dividing cells that are insensitive to androgen but sensitive to other proliferative signals such as those of the MET signaling pathway (32) (Fig. 5).

Notably, the mouse Yap gene copy number was significantly increased in a mouse model of prostate cancer driven by PTEN/p53 loss (33). Yap was the top ranked gene amplified by whole genome screening in this model (33) (Fig. 6). As PTEN and p53 are frequently mutated or inactivated in several cancers, particularly in prostate cancer, this finding suggests that YAP may act as a master regulator in prostate cancer upon oncogenic insults stimulation. Furthermore, YAP may provide feedback regulation through the YAP/PTEN axis (Fig. 4). However, the detailed mechanism of this feedback loop and its clinical relevance must be further investigated.

\section{Implications for clinical targeted therapy}

As aforementioned, YAP is involved in the control of proliferation, and is regulated by different mechanisms. Due to its very important role in tumorigenesis, this protein could be used as a target for novel avenues of cancer therapy. A recent study has suggested several approaches to suppress the activity of YAP in cancer cells with increased cell proliferation rate (34). One of the molecules that competes for YAP activity in the nucleus is VGLL4 (34). This molecule functions as an antagonist and competes with YAP for binding to TEAD family members, and thus can inhibit the activity of YAP. More precisely, VGLL4's tandem Tondu domains inhibit the activity of YAP (33). YAP plays a major role in the formation of HCC (35). In addition, small interfering RNA-lipid nanoparticles, which target and inactivate YAP protein, can decrease the levels of activated YAP protein (35). This causes HCC tumor regression and restores hepatocyte differentiation (35).

Currently, numerous single treatments using anticancer drugs have little effect on cancer cells growth. By contrast, the combination of several drugs is able to cause cancer cells to stop proliferating and induce apoptosis (36). As an example, the combination of the drugs trametinib and vemurafenib, which inhibit regulatory proteins in the mitogen-activated protein kinase signaling pathway, promotes cancer cells to stop proliferating, but still is not sufficient to cause tumor cell death (37). The introduction of RNA interference specific to YAP, which indirectly induces the expression of the pro-survival gene Bcl-extra large, reduces cell proliferation and induces cell apoptosis (36). Thus, YAP protein plays a major role in drug resistance through the regulation of the expression of genes responsible for cancer cell survival. Therefore, targeting YAP may sensitize cancer cells to chemotherapy drugs.

In summary, YAP, as a downstream target of the Hippo signaling pathway, can crosstalk with various biological signaling pathways. Directly targeting of YAP signaling would be a novel avenue for the treatment of aggressive cancer and reversing drug resistance.

\section{References}

1. Lorenzetto E, Brenca M, BoeriM, VerriC,Piccinin E, Gasparini P, Facchinetti F, Rossi S, Salvatore G, Massimino M, et al: YAP1 acts as oncogenic target of 11q22 amplification in multiple cancer subtypes. Oncotarget 5: 2608-2621, 2014.

2. Shen Z and Stanger BZ: YAP regulates S-Phase entry in endothelial cells. PLoS One 10: e0117522, 2015.

3. Zhao B, Li L, Lei Q and Guan KL: The Hippo-YAP pathway in organ size control and tumorigenesis: An updated version. Genes Dev 24: 862-874, 2010.

4. Kanai F, Marignani PA, Sarbassova D, Yagi R, Hall RA, Donowitz M, Hisaminato A, Fujiwara T, Ito Y, Cantley LC and Yaffe MB: TAZ: A novel transcriptional co-activator regulated by interactions with 14-3-3 and PDZ domain proteins. EMBO J 19: 6778-6791, 2000.

5. Shimomura T, Miyamura N, Hata S, Miura R, Hirayama J and Nishina H: The PDZ-binding motif of Yes-associated protein is required for its co-activation of TEAD-mediated CTGF transcription and oncogenic cell transforming activity. Biochem Biophys Res Commun 17: 917-931, 2014.

6. Espanel X and Sudol M: Yes-associated protein and p53-binding protein-2 interact through their WW and SH3 domains. J Biol Chem 276: 14514-14523, 2001.

7. Moroishi T, Hansen CG and Guan KL: The emerging roles of YAP and TAZ in cancer. Nat Rev Cancer 276: 73-79, 2015.

8. Zhou X, Wang Z, Huang W and Lei QY: G protein-coupled receptors: Binding the gap from the extracellular signals to the Hippo pathway. Acta Biochim Biophys Sin (Shanghai) 47: 10-15, 2015.

9. Azzolin L, Panciera T, Soligo S, Enzo E, Bicciato S, Dupont S, Bresolin S, Frasson C, Basso G, Frasson C, et al: YAP/TAZ incorporation in the $\beta$-catenin destruction complex orchestrates the Wnt response. Cell 158: 157-170, 2014.

10. Mitamura T, Watari H, Wang L, Kanno H, Kitagawa M, Hassan MK, Kimura T, Tanino M, Nishihara H, Tanaka S and Sakuragi N: MicroRNA 31 functions as an endometrial cancer oncogene by suppressing Hippo tumor suppressor pathway. Mol Cancer 13: 97, 2014.

11. Zhang L, Tang F, Terracciano L, Hynx D, Kohler R, Bichet S, Hess D, Cron P, Hemmings BA, Hergovich A and Schmitz-Rohmer D: NDR functions as a physiological YAP1 kinase in the intestinal epithelium. Curr Biol 25: 296-305, 2015.

12. Basu S, Totty NF, Irwin MS, Sudol M and Downward J: Akt phosphorylates the Yes-associated protein, YAP, to induce interaction with 14-3-3 and attenuation of p73-mediated apoptosis. Mol Cell 11: 11-23, 2003.

13. Llado V, Nakanishi Y, Duran A, Reina-Campos M, Shelton PM, Linares JF, Yajima T, Campos A, Aza-Blanc P, Leitges M, et al: Repression of intestinal stem cell function and tumorigenesis through direct phosphorylation of $\beta$-catenin andYap by PKC $\zeta$. Cell Rep pii: S2211-1247, 2015. 
14. Oudhoff MJ, Freeman SA, Couzens AL, Antignano F, Kuznetsova E, Min PH, Northrop JP, Lehnertz B, Barsyte-Lovejoy D, Vedadi M, et al: Control of the hippo pathway by Set7-dependent methylation of Yap. Dev Cell 26: 188-194, 2013.

15. Hergovich A and Hemmings BA: Mammalian NDR/LATS protein kinases in hippo tumor suppressor signaling. Biofactors 35: 338-345, 2009.

16. Sorrentino G, Ruggeri N, Specchia V, Cordenonsi M, Mano M, Dupont S, Manfrin A, Ingallina E, Sommaggio R, Vedadi M, et al: Metabolic control of YAP and TAZ by the mevalonate pathway. Nat Cell Biol 16: 357-366, 2014.

17. Low BC, Pan CQ, Shivashankar GV, Bershadsky A, Sudol M and Sheetz M: YAP/TAZ as mechanosensors and mechanotransducers in regulating organ size and tumor growth. FEBS Lett 588: 2663-2670, 2014.

18. Halder G, Dupont S and Piccolo S: Transduction of mechanical and cytoskeletal cues by YAP and TAZ. Nat Rev Mol Cell Biol 13: 591-600, 2012

19. Mana-Capelli S, Paramasivam M, Dutta S and McCollum D: Angiomotins link F-actin architecture to Hippo pathway signaling. Mol Biol Cell 25: 1676-1685, 2014.

20. Johnson R and Halder G: The two faces of Hippo: Targeting the Hippo pathway for regenerative medicine and cancer treatment. Nat Rev Drug Discov 13: 63-79, 2014

21. Harvey KF, Zhang X and Thomas DM: The Hippo pathway and human cancer. Nat Rev Cancer 13: 246-257, 2013.

22. Mori M, Triboulet R, Mohseni M, Schlegelmilch K, Shrestha K, Camargo FD and Gregory RI: Hippo signaling regulates Microprocessor and links cell density-dependent miRNA biogenesis to cancer. Cell 156: 893-906, 2014

23. Chaulk SG, Lattanzi VJ, Hiemer SE, Fahlman RP and Varelas X: The Hippo pathway effectors TAZ/YAP regulate dicer expression and microRNA biogenesis through Let-7. J Biol Chem 289: 1886-1891, 2014

24. Tumaneng K, Schlegelmilch K, Russell RC, Yimlamai D, Basnet H, Mahadevan N, Fitamant J, Bardeesy N, Camargo FD and Guan KL: YAP mediates crosstalk between the Hippo and PI (3)K-TOR pathways by suppressing PTEN via miR-29. Nat Cell Biol 14: 1322-1329, 2012

25. Fausti F1, Di Agostino S, Cioce M, Bielli P, Sette C, Pandolfi PP, Oren M, Sudol M, Strano S and Blandino G: ATM kinase enables the functional axis of YAP, PML and p53 to ameliorate loss of Werner protein-mediated oncogenic senescence. Cell Death Differ 20: 1498-1509, 2013
26. Xie Q, Chen J, Feng H, Peng S, Adams U, Bai Y, Huang L, Li J, Huang J, Meng S, et al: YAP/TEAD-mediated transcription controls cellular senescence. Cancer Res 73: 3615-3624, 2013.

27. Kapoor A, Yao W, Ying H, Hua S, Liewen A, Wang Q, Zhong Y, Wu CJ, Sadanandam A, Hu B et al: Yap1 activation enables bypass of oncogenic Kras addiction in pancreatic cancer. Cell 158: 185-197, 2014.

28. Shao DD, Xue W, Krall EB, Bhutkar A, Piccioni F, Wang X, Schinzel AC, Sood S, Rosenbluh J, Kim JW, et al: KRAS and YAP1 converge to regulate EMT and tumor survival. Cell 158: 171-184, 2014.

29. Greten FR: YAP1 takes over when oncogenic K-Ras slumbers. Cell 158: 11-12, 2014.

30. Alptekin A, Abali GK, Wang Q and Bekir C: 'Regulation of androgenic signaling by yes-associated protein, YAP, in prostate cancer cells'. Cancer Res 73 (8 Supplement): 759, 2013.

31. Zhang L, Yang S, Chen X, Stauffer S, Yu F, Lele SM, Fu K, Datta K, Palermo N, Chen Y and Dong J: The hippo pathway effector, YAP, regulates motility, invasion and castration-resistant growth of prostate cancer cells. Mol Cell Biol 35: 1350-1362, 2015.

32. Xie Y, Lu W, Liu S, Yang Q, Carver BS, Li E, Wang Y, Fazli L, Gleave $M$ and Chen Z: Crosstalk between nuclear MET and SOX9/ $\beta$-catenin correlates with castration-resistant prostate cancer. Mol Endocrinol 28: 1629-1639, 2014.

33. Wanjala J, Taylor BS, Chapinski C, Hieronymus H, Wongvipat J, Chen Y, Nanjangud GJ, Schultz N, Xie Y, Liu Sr, et al: Identifying actionable targets through integrative analyses of GEM model and human prostate cancer genomic profiling. Mol Cancer Ther 14: 278-288, 2015.

34. Jiao S, Wang H, Shi Z, Dong A, Zhang W, Song X, He F, Wang Y, Zhang Z, Wang W, et al: A peptide mimicking VGLL4 function acts as a YAP antagonist therapy against gastric cancer. Cancer Cell 25: 166-180, 2014.

35. Fitamant J, Kottakis F, Benhamouche S, Tian HS, Chuvin N, Parachoniak CA, Nagle JM, Perera RM, Lapouge M, Deshpande V, et al: YAP inhibition restores hepatocyte differentiation in advanced HCC, leading to tumor regression. Cell Rep pii: S2211-S1247, 2015

36. Lin L, Sabnis AJ, Chan E, Olivas V, Cade L, Pazarentzos E, Asthana S, Neel D, Yan JJ, Lu X et al: The Hippo effector YAP promotes resistance to RAF- and MEK-targeted cancer therapies. Nat Genet 47: 250-256, 2015.

37. Eroglu Z and Ribas A: Combination therapy with BRAF and MEK inhibitors for melanoma: Latest evidence and place in therapy. Ther Adv Med Oncol 8: 48-56, 2016. 